Review

\title{
MicroRNA-92a: The Administrator of Certain Diseases
}

\author{
${ }^{1 \#}$ Zhiyuan Sun, ${ }^{2}$ Qing Xu, ${ }^{1}$ Xiaoyi Tian, ${ }^{1}$ Yingjie Yang, ${ }^{3}$ Qinglu Wang and ${ }^{1 *}$ Xuewen Tian \\ ${ }^{1}$ Shandong Sport University, Jinan, Shandong Province, China 250102 \\ ${ }^{2}$ Shandong Sports Rehabilitation Research Center, Department of Rehabilitation Medicine, \\ 3008 Fengming Road, Licheng District, Jinan City, Shandong Province, China 250012 \\ ${ }^{3}$ Key Laboratory of Biomedical Engineering and Technology of Shandong High School, \\ Qilu Medical University, Zibo, Shandong Province, China 255213
}

\section{Article history}

Received: 27-03-2020

Revised: 14-04-2020

Accepted: 19-05-2020

Corresponding Author:

Xuewen Tian

Shandong Sport University,

Jinan, Shandong Province,

China 250102

Email: xuewentian1978@163.com

\begin{abstract}
MicroRNA-92a (miR-92a) is an evolutionarily conserved noncoding small RNA that can regulate gene expression after transcription. Previous studies have found that miR-92a is overexpressed in many tumors and can regulate numerous tumor suppressor genes negatively, with relevant effects on the development of different tumors, by regulating the DUSP10/c-Jun N-terminal kinase (JNK), phosphatase and tensin homologs (PTEN)/AKT, Wnt and EP4/Notch1 signaling axes. MiR-92a also promotes the proliferation and migration of vascular smooth muscle cells (VSMCs) through the Rhoassociated coiled-coil-forming kinase/myosin light chain kinase signaling pathway and inhibits VSMC apoptosis through the MKK4/JNK signaling pathway. Moreover, miR-92a affects endothelial functions; mediates endothelial dysfunction in chronic kidney diseases; mediates THBS1 inhibition; promotes the migration, proliferation and angiogenesis of neighboring endothelial cells (ECs); mediates the Nrf2/KEAP1/ARE signaling pathway to regulate vascular endothelial aging; and is involved in immune responses to activate ECs. This review summarizes the potential role and pathogenic mechanism of the miR-92a gene in certain diseases to provide possible new treatment options.
\end{abstract}

Keywords: microRNA-92a, Cancer, Signaling Pathway, Endothelial Damage, Vascular Smooth Muscle Cell

\section{Introduction}

MicroRNAs (miRNAs) are small (19-24 nt) singlestranded noncoding RNAs that regulate messenger RNA (mRNA) translation and stability by binding to the 3'Untranslated Region (UTR) of target genes (Sun and Lai, 2013; Ameres and Zamore, 2013). Numerous studies have demonstrated that miRNAs play critical roles in various biological processes, such as cell proliferation and apoptosis, glucose and lipid metabolism and infection and immune responses (Szabo and Bala, 2013; Pritchard et al., 2012; Rottiers and Naar, 2012). In addition, the function of miRNAs in human tumorigenesis has been well established, which can add new insights into diagnosis and prognosis procedures (Ling et al., 2013). The miR-17-92 cluster encodes six miRNAs, namely, miR-17, miR-18a, miR-19a, miR-19b, miR-20a and miR-92a, which are located in the coding region of the open reading frame of the $C 13$ or $f 25$ gene.
The human miR-17-92 cluster gene is mapped to chromosome 13q31 (Rao et al., 1998; Ota et al., 2004; Tagawa and Seto, 2005). MicroRNA-92a (miR-92a) is a member of the miR-17-92 cluster and an evolutionarily conserved noncoding small RNA that can regulate gene expression at the post-transcriptional level. In other words, one miRNA generally targets the 3'-UTR of various mRNAs involved in different steps of one precise metabolic/signaling pathway. Moreover, microRNA-92a is an oncogenic (Tsuchida et al., 2011; Wang et al., 2016) as well as a tumor suppressor gene (Shin et al., 2018; Smith et al., 2015). A change in the level of a key miR-92a affects the individual steps of a pathway; thus is promoted or suppressed (Wang et al., 2019) depending on the cancer model. Although the functions of most identified miRNAs have yet to be determined, they are considered as potential biomarkers for several human diseases and cancers (Calin et al., 2004; Volinia et al., 2006). 


\section{Relationship Between miR-92a and Tumors}

\section{Expression and Targeting of miR-92a in Different} Tumors

The ability of miR-92a expression regulation to influence cell proliferation may prove to be a new mechanism for preventing and treating tumors. In addition, miR-92a has the advantage of being stable, noninvasive, convenient and highly sensitive as a biomarker for the early diagnosis of cancer (Yang et al., 2014a). MiR-92a is overexpressed in many tumors and can negatively regulate numerous tumor suppressor genes (Tsuchida et al., 2011), including the downregulated target genes of the von Hippel-Lindau tumor suppressor, farnesoid $\mathrm{X}$ receptor and cadherin1 (Scapoli et al., 2010; Yu et al., 2013; Si et al., 2013). Previous studies have shown that miR-92a is upregulated in the development of several tumors, including in cervical, colon, gastric, oral, breast and lung cancer (Tsuchida et al., 2011; Scapoli et al., 2010; Yu et al., 2013; Si et al., 2013; Valera et al., 2011; Al-Nakhle et al., 2010; Hayashita et al., 2005). Furthermore, miR-92a promotes cancer cell proliferation and survival through several mechanisms, such as downregulation of Estrogen Receptor beta (ER $\beta$ ), Phosphatase and Tensin Homologs (PTEN) and BH3-protein (Tsuchida et al., 2011; Yu et al., 2013; Al-Nakhle et al., 2010). The decreased expression of miR-92a in cancer stem cells leads to the high expression levels of target molecules, that is, integrin $\alpha \mathrm{V}$ and $\alpha 5$ subunits, which in turn enhances TGF- $\beta$ activation, as proven by increased phosphorylation of SMAD2 (Shidal et al., 2019).

MiR-92a and Signaling Pathways that Regulate Tumorigenesis and Development

MiR-92a Promotes Pancreatic Cancer Cell Proliferation Via DUSP10/c-Jun N-terminal Kinase (JNK) Signaling Axis

Pancreatic cancer is one of the most common causes of tumor-related deaths in the world (He et al., 2014). Rapid proliferation is the most important characteristic of cancer cells. JNK signaling is a well-known signaling pathway that regulates the formation and development of cancers and is related to oncogenic transformation (Takahashi et al., 2013; Nateri et al., 2005; Yunoki et al., 2013). MiR-92a enhances the activation of the JNK signaling pathway by directly targeting the JNK signaling inhibitor DUSP10. DUSP10 is responsible for miR-92a-induced JNK signaling and cell proliferation. Overall, the miR-92a/DUSP10/JNK signaling pathway plays an important role in regulating the proliferation of pancreatic cancer cells (He et al., 2014) (Fig. 1).

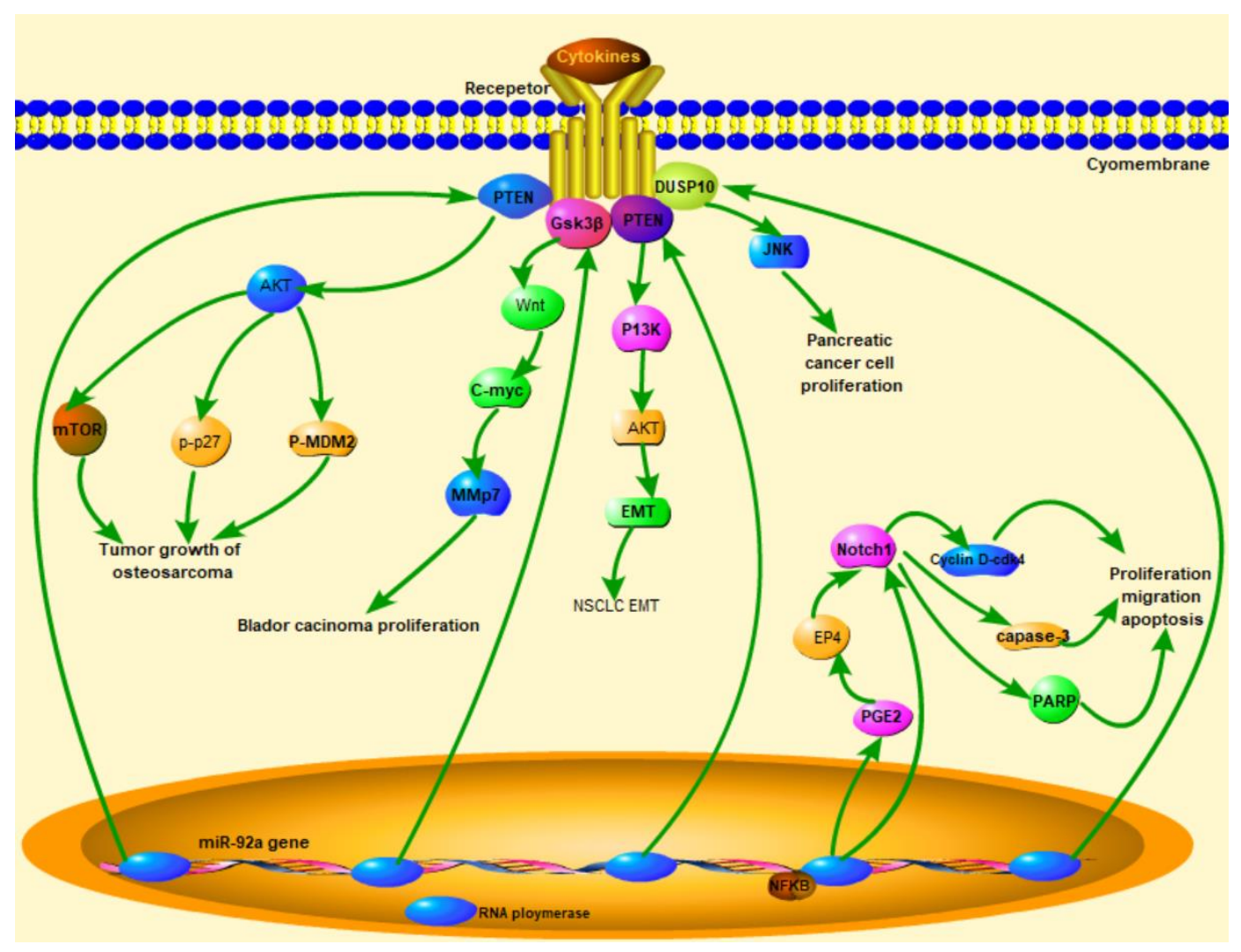

Fig. 1: Effect of miR-92a on cancer cell signaling axis 
Role of miR-92a and PTEN-Related Signaling Pathways in Tumorigenesis and Development

PTEN, which is a well-known tumor suppressor, is the direct downstream target of miR-92a in osteosarcoma, nasopharyngeal carcinoma and Non-Small Cell Lung Carcinoma (NSCLC) (Xiao et al., 2017; Zhang et al., 2016; Lu et al., 2017). Moreover, PTEN, which is a versatile protein, can inversely regulate PI3K/AKT/Epithelial Mesenchymal Transition (EMT) signaling in NSCLC (Scapoli et al., 2010) (Fig. 1). The overexpression of miR-92a enhances EMT-related protein levels, promotes EMT in NSCLC metastasis, facilitates the migration and invasion of NSCLC cells in vitro and increases tumor growth in vivo (Lu et al., 2017). Furthermore, the PTEN/AKT pathway mediates the phosphorylation of p27 (Thr157), AKT (Ser473) and MDM2 (Ser166) (Xie et al., 2016) (Fig. 1). PTEN promotes OS proliferation, inhibits apoptosis and facilitates transfer in NPC by regulating the expression and phosphorylation of the aforementioned proteins (Xiao et al., 2017; Lu et al., 2017). Furthermore, miR92a plays an oncogenic role in OS, NPC and NSCLC by targeting the PTEN signaling pathway.

MiR-92a Promotes Invasion and Chemoresistance by Targeting GSK3 $\beta$ and Activating Wnt Signaling in Bladder Cancer Cells

Prognosis for bladder cancer lies in its histological subtype, tumor size, lymph node metastasis and distant metastasis (Reddy et al., 2015; Szarvas et al., 2012; Yang et al., 2012; Wang et al., 2016). Luciferase reporter assays reveal that miR-92a can directly bind to the $3^{\prime}$-UTR of GSK3 $\beta$, thereby demonstrating that GSK3 $\beta$ is a direct target of miR-92a (Wang et al., 2016). Additionally, depleted GSK3 $\beta$ replace the role of miR92a in its downstream protein cyclin D1 and MMP7. Given that cyclin D1, c-myc and MMP7 are Wnt target proteins, miR-92a binds directly to the 3'-UTR region of GSK $3 \beta$ to promote proliferation, invasion and Wnt/c-myc/MMP7 signaling in bladder cancer cells (Wang et al., 2016) (Fig. 1).

\section{MiR-92a Suppresses Proliferation and Induces Apoptosis by Targeting EP4/Notch1 Axis in Gastric Cancer}

Notch signaling activation has been proven in gastric cancer growths and has a high expression level in human gastric cancer tissues (Yeh et al., 2009). The expression of mir-92a in primary tumors in patients with gastric cancer can be verified via real-time PCR. NF-кB expression is negatively regulated with miR-92 levels in gastric tissues, whereas transfection with NF- $\mathrm{kB}$ siRNA (p50 and p65) increases miR-92 expression in gastric cancer (Shin et al., 2018). Notch signaling has been implicated in various carcinogenesis, including gastric cancer. The phosphorylation of STAT3 and Twistpromoted gastric cancer progression is regulated by the Notch1 receptor intracellular domain (Hsu et al., 2012). Moreover, Prostaglandin E2 (PGE2) plays a crucial role in cancer initiation and progression through its receptors (EP receptor). PGE receptors comprise four G-proteincoupled cell surface receptors, namely EP1, EP2, EP3 and EP4, for signal transduction. Strong evidence shows that PGE2 and its receptors are implicated in the carcinogenesis of different types of tumors, including gastric cancer. Thus, miR-92a has been suggested to regulate cell proliferation and cell invasion through the EP4/Notch1 signaling pathway (Shin et al., 2018) (Fig. 1).

\section{MiR-92a and Signaling Pathways Regulate Vascular Smooth Muscle Cells (VSMCs)}

MiR-92a Promotes VSMC Proliferation and Migration through Rho-Associated Coiled-CoilForming Kinase (ROCK)/Myosin Light Chains (MLCK) Signaling Pathway

Endothelial Cells (ECs) and VSMCs are the main cell types within the vasculature and are closely related in terms of structure and function. ECs that cover the interior surface of blood play an important role in the regulation of vascular tone by releasing vasoactive agents that control VSMC proliferation or migration (Zhao et al., 2012). PDGF-BB, which is found in atherosclerotic lesions, is a well-known potent mitogen and chemoattractant for VSMCs. Moreover, PDGF-BB can activate ROCK and MLCK (Xiong et al., 2017), which regulate the phosphorylation of MLCs (Zhou et al., 2011). The phosphorylation of MLCs promotes cell contraction and cell motility, thereby generating changes in the actin cytoskeleton (Shimokawa and Rashid, 2007). In vitro results indicate that MLCK and miR-92a share the same signaling pathway. The transfection of miR-92a mimics can partially restore the effect of the deficiency of MLCK and antagonize the effect of Y27632 (an inhibitor of ROCK) on the downregulation of VSMC activities (Wang et al., 2019). ML-7 increases the expression of Kruppel-like factor 4 (KLF4, which is an miR-92a target) (Loyer et al., 2014) and siRNA-KLF4 increases the activity levels of VSMCs. The inhibition of either MLCK or ROCK enhances KLF4 expression consistently. Moreover, ROCK/MLCK upregulates miR92a expression in VSMCs through Signal Transducer and Activator of Transcription 3 (STAT3) activation. In summary, the activation of ROCK/STAT3 and/or MLCK/STAT3 may upregulate miR-92a expression, which subsequently inhibits KLF4 expression and promotes the PDGF-BB-mediated proliferation and migration of VSMCs (Wang et al., 2019) (Fig. 2). 


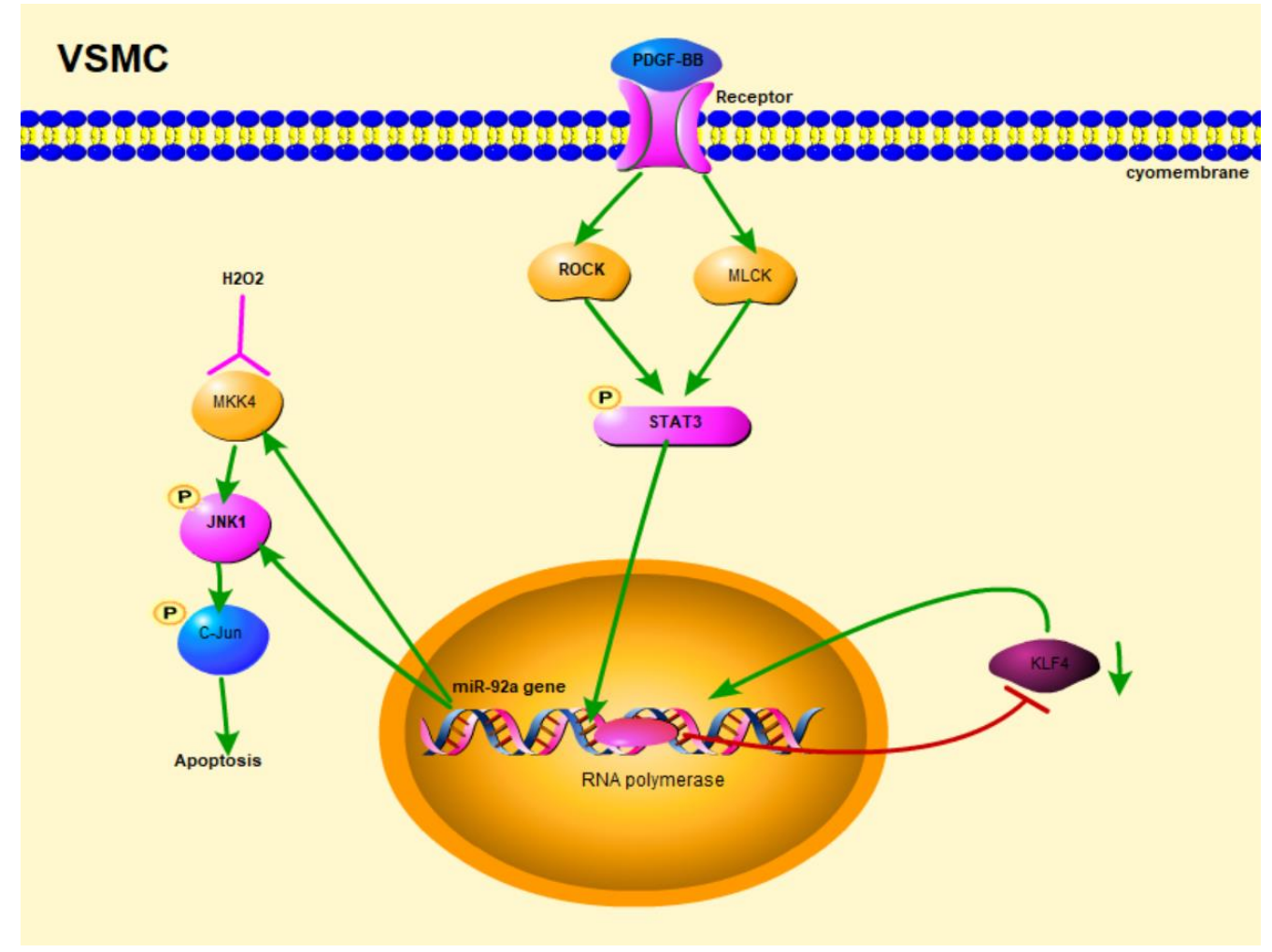

Fig. 2: Effect of miR-92a on VSMC signaling axis

\section{MiR-92a Inhibits Apoptosis of VSMCs Through MKK4/JNK Signaling Pathway}

The apoptosis of VSMCs is believed to be triggered by oxidative stress that is present in diseased vascular milieu (Loyer et al., 2014). The JNK pathway plays a crucial role in VSMC apoptosis in atherosclerotic plaque instability and rupture induced by oxidative stress (Loyer et al., 2014). Given that the JNK1 pathway is involved in VSMC apoptosis induced by oxidative stress (Tchivilev et al., 2008) and MKK4 and JNK1 are identified as target genes for miR-92a, the present study uses a luciferase reporter assay to show that miR-92a regulates the expression of MKK4 by targeting its 3'UTR (Lai et al., 2013). The overexpression of miR-92a reduces the level of the MKK4 protein by $30 \%$ and that of the p54 JNK1 protein by 20\% (Zhang et al., 2014). Moreover, MKK4 and JNK1 are downregulated by miR92a, thereby inhibiting oxidative stress-induced VSMC apoptosis (Zhang et al., 2014) (Fig. 2).

\section{MiR-92a and Signaling Pathways Regulate Endothelial Damage}

\section{MiR-92a Mediates Endothelial Dysfunction in Chronic Kidney Disease (CKD)}

$\mathrm{CKD}$ is an independent risk factor for cardiovascular disease (CVD) (Jourde-Chiche et al.,
2006). MiR-92a is induced by oxidative stress in ECs (Chen et al., 2015) and involved in angiogenesis and atherosclerosis (Loyer et al., 2014). Mechanistically, miR-92a targets the 39 UTRs of mRNA-encoding sirtuin 1 (SIRT1), KLF2 and KLF4, all of which positively regulate eNOS-derived NO (Fang and Davies, 2012). In ECs, miR-92a targets SIRT1, KLF2 and KLF4, which are essential for endothelial homeostasis and functions (Chen et al., 2015). CKDinduced miR-92a potentiates endothelial dysfunction and contributes to CVD. Uremic toxins (e.g., IS) in CKD increase the oxidative stress burden in vascular endothelium. In turn, oxidative stress-induced miR-92a can lead to endothelial dysfunction and is released by MPs into the circulation, which can impair endothelial homeostasis further and increase CVD risk (Shang et al., 2017) (Fig. 3).

\section{Regulation of miR-92a in Vascular Endothelial Aging by Mediating Nrf2-KEAP1-ARE Signal Pathway}

When a body is under oxidative stress, the overproduction of free oxygen species may exceed the clearance potency of the body, thereby leading to an imbalance in the oxidation/antioxidation system and causing further tissue inflammation and injury (Zhang et al., 2015). SOD is an important antioxidase in the body for clearing ROS and plays a critical role in oxidation/antioxidation homeostasis (Liu et al., 2015). 


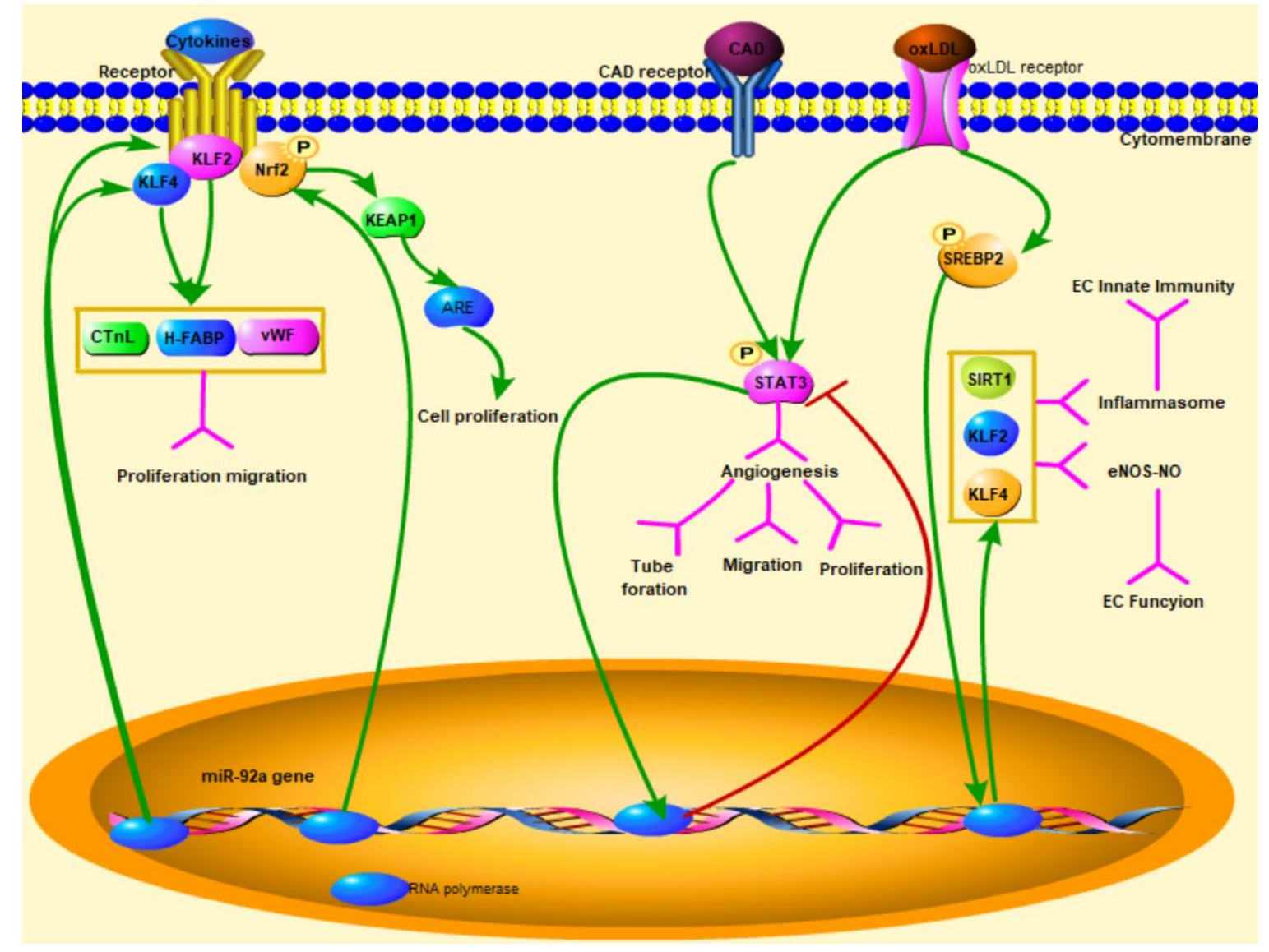

Fig. 3: Effect of miR-92a on EC signaling axis

MPO cannot penetrate a membrane alone but can be released abundantly under cell injury or death (Yang et al., 2014b). Moreover, the downregulation of miR-92a facilitates SOD expression in aging vascular ECs and decreases ROS and MPO, thereby further illustrating that miR-92a downregulation can mediate oxidation/antioxidation balance, thereby decreasing caspase- 3 activity and facilitating proliferation and aging cells. Nrf2 is a transcription factor with cellprotecting effects (Jiang et al., 2016). The Nrf2KEAP1-ARE signal pathway is a critical endogenous antioxidation signal pathway in the body and can resist internal/external oxidation and chemical substances by mediating redox balance, inhibiting oxidative stress further and exerting defense effects (Chatterjee et al., 2016; Menegon et al., 2016) (Fig. 3 ). Moreover, the downregulation of miR-92a can inhibit KEAP1 expression substantially and facilitate Nrf2 and ARE expression, thereby indicating that the Nrf2-KEAP1-ARE signal pathway plays a critical role in aging oxidative stress response. The downregulation of miR-92a can affect the aging process of vascular ECs by mediating the Nrf2KEAP1-ARE signal pathway (Liu et al., 2017).
MiR-92 Promotes the Migration, Proliferation and Angiogenesis of Neighboring ECs by Mediating THBS1 Inhibition

As an independent risk factor of atherosclerosis, Low-Density Lipoprotein (LDL), specifically its oxidized form (i.e., oxLDL), plays a key role in endothelial dysfunction and atherogenesis (Pirillo et al., 2013). OxLDL induces the expression of miR-92 and promotes the packing of miR-92 from ECs into the EMV. Under atherosclerotic conditions, the expression of miR92 in ECs and the EMV is upregulated in a STAT3dependent manner. The EMV released from maternal ECs contains miR-92, which is transferred to neighboring ECs, thereby promoting the migration, proliferation and angiogenesis of adjacent ECs through the inhibition of miR-92-mediated THBS1 (Liu et al., 2019) (Fig. 3).

\section{MiR-92a Activates EC Immunity}

Given the positive effect of SIRT1, KLF2 and KLF4 on NO bioavailability, the finding that SREBP2-miR92a suppresses the expression of SIRT1, KLF2 and KLF4 reveals a post-transcriptional mechanism through 
which oxidative stress diminishes NO bioavailability, thereby resulting in endothelial dysfunction (Chen et al., 2015). Oxidative stress induces SREBP2 and miR-92a in ECs as well as SREBP2-activated miR-92a. Oxidative stress-induced miR-92a-targeting SIRT1, KLF2 and KLF4 increase endothelial innate immunity but decrease NO bioavailability. Moreover, miR-92a levels are negatively correlated with patient EC functions (Fang and Davies, 2012; Wu et al., 2011; Chen et al., 2010) (Fig. 3). SREBP2-induced miR-92a targets key molecules in endothelial homeostasis, including SREBP1, KLF2 and KLF4, thereby leading to NOD-like receptor family, pyrin domain containing 3 inflammasome activation and eNOS inhibition. In ECspecific SREBP2 transgenic mice, locked nucleic acid (LNA)-modified antisense miR-92a (LNA-92a) attenuates inflammasome, improves vasodilation and ameliorates Ang II-induced and aging-related atherogenesis (Chen et al., 2015).

\section{Conclusion and Perspectives}

MiR-92a binds different target genes to regulate numerous signaling pathways that can alter cancer cells and plays an important role in tumorigenesis, development and metastasis. Given that miRNAs are stable in tumor tissues and plasma, miR-92a is expected to make a breakthrough in early tumor diagnosis and tumor gene therapy research. MiR-92a promotes the proliferation and migration of VSMCs and inhibits the apoptosis of VSMCs effectively by regulating their related signaling pathways. Moreover, miR-92a mediates endothelial dysfunction in CKD, regulates vascular endothelial aging and promotes migration, proliferation and angiogenesis in adjacent ECs. These processes can in turn lead to endothelial damage in the human body, thereby reducing inflammation and improving vasodilation and atherosclerosis by regulating the expression level of miR-92a. Furthermore, miR-92a can be used as a biomarker and potential therapeutic target for the diagnosis of diseases. Overall, the miR-92a can participate in the regulation of multiple signaling pathways and play a potential role in certain diseases. Abnormal changes in different signaling pathways cause different diseases and also induce miR-92a abnormal expression (up- or down-). Different pathways correspond to different disease states or/and physiological environments. Under this specific state and environment, it is only possible to activate a related signaling pathway and other signaling pathways may not be activated. With further research, the differential expression of the miR-92a gene in cells, stem cells, or drug-resistant cells as well as its mechanism may provide a new direction for disease studies. Further research should be conducted in the future to discover the target of miR-92a and its role in different disease signaling pathways and to understand the pathogenic mechanism behind its activity.

\section{Acknowledgement}

This work was supported by grants from the National Natural Science Foundation of China (31701042) and the Taishan Scholars Program of Shandong Province (tsqn201909148) and the Natural Scientific Foundation of Shandong Province, China (ZR2018MH038) and Zibo Platform for Gene Editing and Cell Application (2018ZBXC010, 2018ZBXC008).

\section{Author's Contributions}

Zhiyuan Sun: Article conception and writing.

Qing Xu: Collection and analysis of literature data.

Xiaoyi Tian and Yingjie Yang: Data collection.

Qinglu Wang: Design and supervise the completion of the project.

Xuewen Tian: Participate in article writing, design and supervise project completion.

\section{Conflict of Interest}

The authors declare that there are no conflicts of interest.

\section{References}

Al-Nakhle, H., P.A. Burns, M. Cummings, A.M. Hanby and T.A. Hughes et al., 2010. Estrogen receptor $\beta 1$ expression is regulated by $\mathrm{miR}-92$ in breast cancer. Cancer Res., 70: 4778-4784.

DOI: 10.1158/0008-5472.CAN-09-4104

Ameres, S.L. and P.D. Zamore, 2013. Diversifying microRNA sequence and function. Nat. Rev. Mol. Cell Biol., 14: 475-488. DOI: 10.1038/nrm3611

Calin, G.A., C.G. Liu, C. Sevignani, M. Ferracin and N. Felli et al., 2004. MicroRNA profiling reveals distinct signatures in B cell chronic lymphocytic leukemias. Proc. Nat. Acad. Sci., 101: 11755-11760. DOI: 10.1073/pnas.0404432101

Chatterjee, N., M. Tian, K. Spirohn, M. Boutros and D. Bohmann, 2016. Keap1-independent regulation of Nrf2 activity by protein acetylation and a BET bromodomain protein. PLoS Genet., 12: e1006072-e1006072.

DOI: 10.1371/journal.pgen.1006072

Chen, Z., I.C. Peng, X. Cui, Y.S. Li and S. Chien et al., 2010. Shear stress, SIRT1 and vascular homeostasis. Proc. Nat. Acad. Sci., 107: 10268-10273. DOI: $10.1073 /$ pnas. 1003833107

Chen, Z., L. Wen, M. Martin, C.Y. Hsu and L. Fang et al., 2015. Oxidative stress activates endothelial innate immunity via Sterol Regulatory Element Binding Protein 2 (SREBP2) transactivation of microRNA-92a. Circulation, 131: 805-814. DOI: $10.1161 /$ CIRCULATIONAHA.114.013675 
Fang, Y. and P.F. Davies, 2012. Site-specific microRNA-92a regulation of Krüppel-like factors 4 and 2 in atherosusceptible endothelium. Arteriosclerosis Thrombosis Vascular Biol., 32: 979-987. DOI: 10.1161/ATVBAHA.111.244053

Hayashita, Y., H. Osada, Y. Tatematsu, H. Yamada and K. Yanagisawa et al., 2005. A polycistronic microRNA cluster, miR-17-92, is overexpressed in human lung cancers and enhances cell proliferation. Cancer Res., 65: 9628-9632.

DOI: 10.1158/0008-5472.CAN-05-2352

He, G., L. Zhang, Q. Li and L. Yang, 2014. miR92a/DUSP10/JNK signalling axis promotes human pancreatic cancer cells proliferation. Biomed. Pharmacotherapy, 68: 25-30.

DOI: 10.1016/j.biopha.2013.11.004

Hsu, K.W., R.H. Hsieh, K.H. Huang, A.F.Y. Li and C.W. Chi et al., 2012. Activation of the Notch1/STAT3/Twist signaling axis promotes gastric cancer progression. Carcinogenesis, 33: 1459-1467. DOI: $10.1093 /$ carcin/bgs 165

Jiang, W.D., B. Qu, L. Feng, J. Jiang and S.Y. Kuang et al., 2016. Histidine prevents cu-induced oxidative stress and the associated decreases in mRNA from encoding tight junction proteins in the intestine of grass carp (Ctenopharyngodon idella). PloS One, 11: e0157001-e0157001.

DOI: 10.1371/journal.pone.0157001

Jourde-Chiche, N., L. Dou, C. Cerini, F. Dignat-George and P. Brunet, 2006. Elevation of circulating endothelial microparticles in patients with chronic renal failure. J. Thromb. Haemost., 4: 566-573. DOI: 10.1111/j.1538-7836.2005.01780.x

Lai, L., Y. Song, Y. Liu, Q. Chen and Q. Han et al., 2013. MicroRNA-92a negatively regulates TollLike Receptor (TLR)-triggered inflammatory response in macrophages by targeting MKK4 kinase. J. Biol. Chem., 288: 7956-7967.

DOI: 10.1074/jbc.M112.445429

Ling, H., M. Fabbri and G.A. Calin, 2013. MicroRNAs and other non-coding RNAs as targets for anticancer drug development. Nat. Rev. Drug Discovery, 12: 847-847. DOI: $10.1038 / \mathrm{nrd} 4140$

Liu, H., H.Y. Wu, W.Y. Wang, Z.L. Zhao and X.Y. Liu et al., 2017. Regulation of miR-92a on vascular endothelial aging via mediating Nrf2-KEAP1-ARE signal pathway. Eur. Rev. Med. Pharmacol. Sci., 21: 2734-2742. PMID: 28678311

Liu, X., Y.Q. Li, C. Hua, S.J. Li and G. Zhao et al., 2015. Oxidative stress inhibits growth and induces apoptotic cell death in human U251 glioma cells via the caspase-3-dependent pathway. Eur. Rev. Med. Pharmacol. Sci., 19: 4068-4075. PMID: 26592828
Liu, Y., Q. Li, M.R. Hosen, A. Zietzer and A. Flender et al., 2019. Atherosclerotic conditions promote the packaging of functional MicroRNA-92a-3p into endothelial microvesicles. Circulat. Res., 124: 575-587. DOI: 10.1161/circresaha.118.314010

Loyer, X., S. Potteaux, A.C. Vion, C.L. Guérin and S. Boulkroun et al., 2014. Inhibition of microRNA-92a prevents endothelial dysfunction and atherosclerosis in mice. Circulat. Res., 114: 434-443.

DOI: 10.1161/CIRCRESAHA.114.302213

Lu, C., Z. Shan, J. Hong and L. Yang, 2017. MicroRNA92a promotes epithelial-mesenchymal transition through activation of PTEN/PI3K/AKT signaling pathway in non-small cell lung cancer metastasis. Int. J. Oncol., 51: 235-244.

Menegon, S., A. Columbano and S. Giordano, 2016. The dual roles of NRF2 in cancer. Trends Molecular Med., 22: 578-593.

DOI: $10.1016 /$ j.molmed.2016.05.002

Nateri, A.S., B. Spencer-Dene and A. Behrens, 2005. Interaction of phosphorylated c-Jun with TCF4 regulates intestinal cancer development. Nature, 437: 281-285. DOI: 10.1038/nature03914

Ota, A., H. Tagawa, S. Karnan, S. Tsuzuki and A. Karpas et al., 2004. Identification and characterization of a novel gene, C13orf25, as a target for 13q31-q32 amplification in malignant lymphoma. Cancer Res., 64: 3087-3095. DOI: 10.1158/0008-5472.CAN-03-3773

Pirillo, A., G.D. Norata and A.L. Catapano, 2013. LOX1, OxLDL and atherosclerosis. Mediators Inflammat. DOI: 10.1155/2013/152786

Pritchard, C.C., H.H. Cheng and M. Tewari, 2012. MicroRNA profiling: Approaches and considerations. Nat. Rev. Genet., 13: 358-369. DOI: $10.1038 / \mathrm{nrg} 3198$

Rao, P.H., J. Houldsworth, K. Dyomina, N.Z. Parsa and J.C. Cigudosa et al., 1998. Chromosomal and gene amplification in diffuse large B-cell lymphoma. Blood J. Am. Society Hematol., 92: 234-240. DOI: 10.1182/blood.V92.1.234.413k22_234_240

Reddy, O.L., J.M. Cates, L.L. Gellert, H.S. Crist and Z. Yang et al., 2015. Loss of FOXA1 drives sexually dimorphic changes in urothelial differentiation and is an independent predictor of poor prognosis in bladder cancer. Am. J. Pathol., 185: 1385-1395. DOI: 10.1016/j.ajpath.2015.01.014

Rottiers, V. and A.M. Naar, 2012. MicroRNAs in metabolism and metabolic disorders. Nat. Rev. Mol. Cell Biol., 13: 239-250. DOI: 10.1038/nrm3313

Scapoli, L., A. Palmieri, L. Lo Muzio, F. Pezzetti and C. Rubini et al., 2010. MicroRNA expression profiling of oral carcinoma identifies new markers of tumor progression. Int. J. Immunopathol. Pharmacol., 23: 1229-1234. DOI: 10.1177/039463201002300427 
Shang, F., S.C. Wang, C.Y. Hsu, Y. Miao and M. Martin et al., 2017. MicroRNA-92a mediates endothelial dysfunction in CKD. J. Am. Society Nephrol., 28: 3251-3261. DOI: 10.1681/ASN.2016111215

Shidal, C., N.P. Singh, P. Nagarkatti and M. Nagarkatti, 2019. MicroRNA-92 expression in CD133+ melanoma stem cells regulates immunosuppression in the tumor microenvironment via integrindependent activation of TGF $\beta$. Cancer Res., 79: 3622-3635. DOI: 10.1158/0008-5472.CAN-18-2659

Shimokawa, H. and M. Rashid, 2007. Development of Rho-kinase inhibitors for cardiovascular medicine. Trends Pharmacol. Sci., 28: 296-302. DOI: 10.1016/j.tips.2007.04.006

Shin, V.Y., M.T. Siu, X. Liu, E.K.O. Ng and A. Kwong et al., 2018. MiR-92 suppresses proliferation and induces apoptosis by targeting EP4/Notch1 axis in gastric cancer. Oncotarget, 9: 24209-24220. DOI: 10.18632/oncotarget.24819

Si, H., X. Sun, Y. Chen, Y. Cao and S. Chen et al., 2013. Circulating microRNA-92a and microRNA-21 as novel minimally invasive biomarkers for primary breast cancer. J. Cancer Res. Clin. Oncol., 139: 223-229. DOI: 10.1007/s00432-012-1315-y

Smith, L., E.W. Baxter, P.A. Chambers, C.A. Green and A.M. Hanby et al., 2015. Down-regulation of miR92 in breast epithelial cells and in normal but not tumour fibroblasts contributes to breast carcinogenesis. PLoS One, 10: e0139698-e0139698. DOI: 10.1371/journal.pone.0139698

Sun, K. and E.C. Lai, 2013. Adult-specific functions of animal microRNAs. Nat. Rev. Genet., 14: 535-548. DOI: $10.1038 / \mathrm{nrg} 3471$

Szabo, G. and S. Bala, 2013. MicroRNAs in liver disease. Nat. Rev. Gastroenterol. Hepatol., 10: 542-552. DOI: 10.1038/nrgastro.2013.87

Szarvas, T., V. László, F. vom Dorp, H. Reis and A. Szendröi et al., 2012. Serum endostatin levels correlate with enhanced extracellular matrix degradation and poor patients' prognosis in bladder cancer. Int. J. Cancer, 130: 2922-2929. DOI: 10.1002/ijc.26343

Tagawa, H. and M. Seto, 2005. A microRNA cluster as a target of genomic amplification in malignant lymphoma. Leukemia, 19: 2013-2016.

DOI: $10.1038 /$ sj.leu.2403942

Takahashi, R., Y. Hirata, K. Sakitani, W. Nakata and H. Kinoshita et al., 2013. Therapeutic effect of c-Jun $\mathrm{N}$-terminal kinase inhibition on pancreatic cancer. Cancer Sci., 104: 337-344. DOI: 10.1111/cas. 12080

Tchivilev, I., N.R. Madamanchi, A.E. Vendrov, X.L. Niu and M.S. Runge, 2008. Identification of a protective role for protein phosphatase $1 \mathrm{c} \gamma 1$ against oxidative stress-induced vascular smooth muscle cell apoptosis. J. Biol. Chem., 283: 22193-22205.

DOI: $10.1074 /$ jbc.M803452200
Tsuchida, A., S. Ohno, W. Wu, N. Borjigin and K. Fujita et al., 2011. miR-92 is a key oncogenic component of the miR-17-92 cluster in colon cancer. Cancer Sci., 102: 2264-2271.

DOI: $10.1111 /$ j.1349-7006.2011.02081.x

Valera, V.A., B.A. Walter, W.M. Linehan and M.J. Merino, 2011. Regulatory effects of microRNA-92 (miR-92) on VHL gene expression and the hypoxic activation of miR-210 in clear cell renal cell carcinoma. J. Cancer, 2: 515-515.

DOI: $10.7150 /$ jca. 2.515

Volinia, S., G.A. Calin, C.G. Liu, S. Ambs and A. Cimmino et al., 2006. A microRNA expression signature of human solid tumors defines cancer gene targets. Proc. Nat. Acad. Sci., 103: 2257-2261. DOI: $10.1073 /$ pnas.0510565103

Wang, H., C. Ke, X. Ma, Q. Zhao and M. Yang et al., 2016. MicroRNA-92 promotes invasion and chemoresistance by targeting GSK $3 \beta$ and activating Wnt signaling in bladder cancer cells. Tumor Biol., 37: 16295-16304. DOI: 10.1007/s13277-016-5460-9

Wang, J., C. Zhang, C. Li, D. Zhao and S. Li et al., 2019. MicroRNA-92a promotes vascular smooth muscle cell proliferation and migration through the ROCK/MLCK signalling pathway. J. Cellular Molecular Med., 23: 3696-3710.

DOI: $10.1111 /$ jcmm.14274

Wu, W., H. Xiao, A. Laguna-Fernandez, G. VillarrealJr and K.C. Wang et al., 2011. Flow-dependent regulation of Krüppel-like factor 2 is mediated by microRNA-92a. Circulation, 124: 633-641.

DOI: 10.1161/CIRCULATIONAHA.110.005108

Xiao, J., W. Yu, K. Hu, M. Li and J. Chen et al., 2017. miR-92a promotes tumor growth of osteosarcoma by targeting PTEN/AKT signaling pathway. Oncol. Rep., 37: 2513-2521. DOI: 10.3892/or.2017.5484

Xie, Y., S. Naizabekov, Z. Chen and T. Tokay, 2016. Power of PTEN/AKT: Molecular switch between tumor suppressors and oncogenes. Oncol., Lett., 12: 375-378.

Xiong, Y., C. Wang, L. Shi, L. Wang and Z. Zhou et al., 2017. Myosin light chain kinase: A potential target for treatment of inflammatory diseases. Frontiers Pharmacol., 8: 292-292.

DOI: $10.3389 /$ fphar.2017.00292

Yang, G.L., L.H. Zhang, J.J. Bo, X.J. Huo and H.G. Chen et al., 2012. Increased expression of HMGB1 is associated with poor prognosis in human bladder cancer. J. Surgical Oncol., 106: 57-61. DOI: $10.1002 /$ jso. 23040

Yang, X., Z. Zeng, Y. Hou, T. Yuan and C. Gao et al., 2014a. MicroRNA-92a as a potential biomarker in diagnosis of colorectal cancer: A systematic review and meta-analysis. PloS One, 9: e88745-e88745. DOI: 10.1371/journal.pone.0088745 
Yang, S., J. Liu and C. Li, 2014b. Intermedin protects against myocardial ischemia-reperfusion injury in hyperlipidemia rats. Genet. Mol. Res., 13: 8309-8319. DOI: 10.4238/2014.October.20.7

Yeh, T.S., C.W. Wu, K.W. Hsu, W.J. Liao and M.C. Yang et al., 2009. The activated Notch1 signal pathway is associated with gastric cancer progression through cyclooxygenase-2. Cancer Res., 69: 5039-5048. DOI: 10.1158/0008-5472.CAN-08-4021

Yu, Y., Y. Zhang and S. Zhang, 2013. MicroRNA-92 regulates cervical tumorigenesis and its expression is upregulated by human papillomavirus-16 E6 in cervical cancer cells. Oncol. Lett., 6: 468-474. DOI: 10.3892/ol.2013.1404

Yunoki, T., A. Kariya, T. Kondo, A. Hayashi and Y. Tabuchi, 2013. The combination of silencing BAG3 and inhibition of the JNK pathway enhances hyperthermia sensitivity in human oral squamous cell carcinoma cells. Cancer Lett., 335: 52-57.

DOI: 10.1016/j.canlet.2013.01.049

Zhang, H., H. Cao, D. Xu and K. Zhu, 2016. MicroRNA-92a promotes metastasis of nasopharyngeal carcinoma by targeting the PTEN/AKT pathway. OncoTargets Therapy, 9: 3579-3579. DOI: 10.2147/OTT.S105470
Zhang, L., M. Zhou, Y. Wang, W. Huang and G. Qin, et al., 2014. miR-92a inhibits vascular smooth muscle cell apoptosis: Role of the MKK4-JNK pathway. Apoptosis, 19: 975-983. DOI: 10.1007/s10495-014-0987-y

Zhang, W., B. Xing, L. Yang, J. Shi and X. Zhou, 2015. Icaritin attenuates myocardial ischemia and reperfusion injury via anti-inflammatory and antioxidative stress effects in rats. Am. J. Chinese Med., 43: 1083-1097. DOI: 10.1142/S0192415X15500627

Zhao, Y., M. Lv, H. Lin, Y. Hong and F.C. Yang et al., 2012. ROCK1 induces ERK nuclear translocation in PDGF-BB-stimulated migration of rat vascular smooth muscle cells. IUBMB Life, 64: 194-202. DOI: $10.1002 /$ iub.598

Zhou, Q., C. Gensch and J.K. Liao, 2011. Rhoassociated Coiled-coil-forming Kinases (ROCKs): Potential targets for the treatment of atherosclerosis and vascular disease. Trends Pharmacol. Sci., 32; 167-173. DOI: 10.1016/j.tips.2010.12.006 